\title{
Comparison of VLBI radio source catalogs
}

\author{
S. Lambert \\ Observatoire de Paris, Systèmes de Référence Temps Espace (SYRTE), CNRS, UPMC, GRGS, Paris, France \\ e-mail: sebastien.lambert@obspm.fr
}

Received 26 June 2014 / Accepted 18 August 2014

\begin{abstract}
Aims. I checked the consistency of recent astrometric radio source catalogs obtained by geodetic very long baseline radio interferometry (VLBI) with the second realization of the International Celestial Reference Frame (ICRF2), released in 2009, which is the most accurate astrometric catalog currently available.

Methods. The catalogs were compared to the ICRF2 in terms of radio source coordinates, global second-degree deformations, and error distribution.

Results. All catalogs were found to be consistent with the ICRF2 within $20 \mu$ as. At high observational rates, the formal error is likely limited to the level of $\sim 10 \mu$ as by correlated-noise errors. The comparison of offsets to ICRF2 against formal errors raised noise floors of the differences between $50 \mu$ as and $100 \mu$ as, and hence no improvement with respect to the ICRF2.

Conclusions. The inconsistencies between catalogs result in differences significantly larger than the accuracy expected for the future realizations of the celestial reference frame. These inconsistencies have to be clarified in the near future in view of the next ICRF realization and accurate linking to reference frames at other frequencies.
\end{abstract}

Key words. astrometry - reference systems

\section{Introduction}

In 2009, the International Astronomical Union (IAU) recommended the adoption of the second realization of the International Celestial Reference Frame (ICRF2), made up of precise coordinates of 3414 extragalactic radio sources observed with very long baseline radio interferometry (VLBI) in geodetic mode at 2 and $8 \mathrm{GHz}$ (Fey et al. 2009). This new fundamental catalog took advantage of recent improvements in the VLBI technique, both at the observational level (new antennas, better data acquisition systems) and at the analysis level (improved analysis methods). The ICRF2 presents a noise floor of 40 microarc seconds ( $\mu$ as). The frame axes are defined by the coordinates of 295 sources and are stable at $10 \mu$ as over 1979-2009.

The maintenance of the ICRF2 is achieved by regularly monitoring the radio sources and producing absolute astrometry catalogs of thousands of quasars. Geodetic VLBI observations and analyses are mainly managed within the International VLBI Service for Geodesy and Astrometry (IVS; Schuh \& Behrend 2012). Some IVS analysis centers regularly submit new catalogs obtained by processing a part of or the entire geodetic VLBI observational data base. The process of validation of these catalogs is then performed in the framework of the International Celestial Reference System Product Center (ICRS-PC) of the International Earth Rotation and Reference Systems Service (IERS), of which one component is hosted at the Paris Observatory.

The data sets are presented in Sect. 2. Then, I propose to investigate the global systematics between a number of recently submitted catalogs (Sect. 3), as well as the consistency of the formal errors with the number of observations and with the offsets to the ICRF2 (Sect. 4).

\section{Data sets}

We consider five recent catalogs submitted to the IVS by different analysis centers. They were established at Geoscience Australia (solution aus2013a); the Federal Agency for Cartography and Geodesy and Institute of Geodesy and Geoinformation of the University of Bonn, Germany (bkg2013a); the NASA Goddard Space Flight Center (GSFC), USA (gsf2012a); the Institute of Applied Astronomy of the Russian Academy of Sciences (iaa2012a); and the Paris Observatory, France (opa2013a). All catalogs were obtained by an ionosphere-free single inversion of VLBI delays accumulated during several thousands of single- and multi-baseline diurnal geodetic sessions at $8 \mathrm{GHz}$. Although aus2013a was processed with OCCAM (Titov et al. 2004) and iaa2012a used QUASAR (Skurikhina et al. 2013), all other solutions were obtained with the Calc/Solve geodetic VLBI analysis software package developed and maintained at GSFC. All these software packages use the state-of-the-art astrometric and geophysical modeling consistent with the IERS Conventions (Petit \& Luzum 2010). Catalogs and exhaustive technical descriptions are available by anonymous ftp to the IVS data center ${ }^{1}$ except for gsf2012a whose information is provided through the server of the GSFC VLBI group ${ }^{2}$.

\footnotetext{
1 ftp://ivsopar.obspm. fr/vlbi/ivsproducts/crf

2 ftp://gemini.gsfc.nasa.gov/solutions/2012a_astro/ 2012a_astro.html
} 
Table 1. Characteristics of the catalogs.

\begin{tabular}{lrrrr}
\hline \hline & \multicolumn{3}{c}{ No. sources } & \\
\cline { 2 - 4 } & Total & ICRF2 & Defining & Mean declination \\
\hline ICRF2 & 3414 & 3414 & 295 & $9.5^{\circ}$ \\
aus2013a & 2949 & 2912 & 291 & $11.3^{\circ}$ \\
bkg2013a & 3294 & 3097 & 287 & $11.9^{\circ}$ \\
gsf2012a & 3708 & 3413 & 295 & $8.4^{\circ}$ \\
iaa2012a & 2943 & 2796 & 292 & $8.7^{\circ}$ \\
opa2013a & 3580 & 3375 & 295 & $9.3^{\circ}$ \\
\hline
\end{tabular}
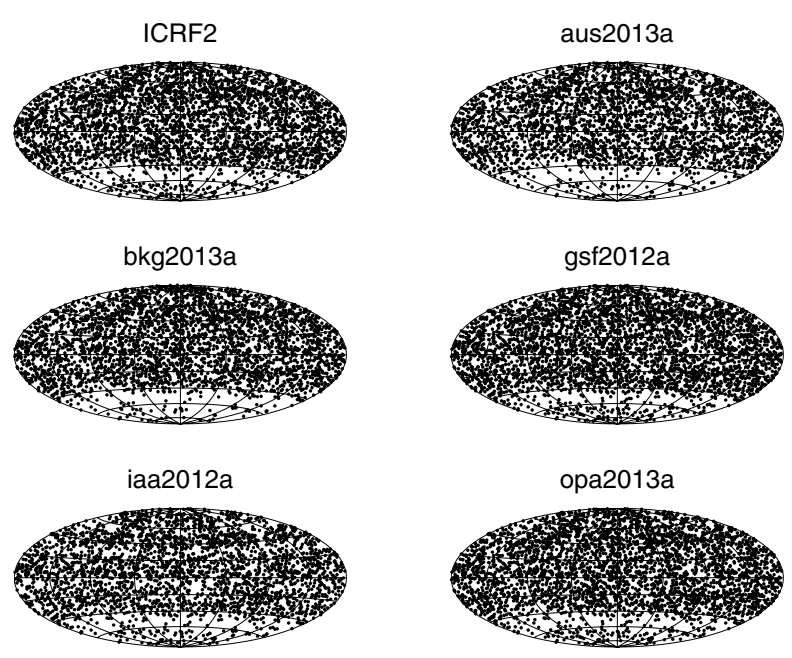

Fig. 1. Catalogs compared in this study.

Nevertheless, if one can reasonably consider that $90 \%$ of the analysis chain is common to all software packages, a number of analysis options are left to the analyst's choice. At the level of accuracy reached nowadays, these variants can have noticeable consequences on the derived VLBI products (Feissel-Vernier 2003; Feissel-Vernier et al. 2005, 2006; Lambert \& Dehant 2007; Heinkelmann \& Tesmer 2013). These options include the choice of the sessions to be analyzed, the exclusion of a part of the data (e.g., because of bad quality), and various aspects relevant to the parameterization (split between global, sessionwise, and arc parameters) and to the constraints (especially those applied globally to the station and radio source coordinates). Moreover OCCAM uses a least squares collocated method while other software packages use classical weighted least squares.

The source distribution for each catalog is shown in Fig. 1. A gap beyond $30^{\circ}$ south shows up in all solutions. Ongoing efforts of the VLBI community are currently filling it in, preparing for the realization of the next generation ICRF (Jacobs et al. 2014). An important point is revealed in Table 1: none of the catalogs provide the coordinates of all the 3414 ICRF2 sources. Only two catalogs provide coordinates for the complete set of the 295 defining sources, very likely because some sessions that were used to generate the ICRF2 in 2009 were not reanalyzed in more recent solutions.

\section{Systematics}

VLBI analysis provides precise relative astrometry. The orientation of the full constellation of radio sources is guaranteed by the no-net rotation (NNR) condition applied to the coordinates of the defining sources that are supposed to be globally non-rotating with respect to the far Universe. To be efficient, the constraint should preferably be applied to the full set of defining sources. A subset of these sources, if not large enough, does not necessarily verify the NNR.

The radio source coordinate difference between catalogs can be modeled by a coordinate transformation that takes into account the global rotation between the catalogs as well as the low-degree deformations. The coordinate transformation recommended by the IERS and in use at the ICRS-PC reads (IERS 1996)

$$
\begin{aligned}
\Delta \alpha & =A_{1} \cos \alpha \sin \delta+A_{2} \sin \alpha \sin \delta-A_{3}+D_{\alpha}\left(\delta-\delta_{0}\right), \\
\Delta \delta & =-A_{1} \sin \alpha+A_{2} \cos \alpha+D_{\delta}\left(\delta-\delta_{0}\right)+B_{\delta},
\end{aligned}
$$

where $\delta_{0}$ is an arbitrary origin of the declination that can be set to zero; $A_{1}, A_{2}$, and $A_{3}$ are rotation angles around the $X, Y$, and $Z$ axes, respectively; $D_{\alpha}$ and $D_{\delta}$ drifts in right ascension and declination as a function of the declination; and $B_{\delta}$ a bias in declination. The transformation parameters estimated over 2294 sources common to all catalogs, of which 286 are ICRF2 defining sources, are displayed in Table 2. The observations were weighted by the inverse of the quadratic sum of the formal errors given by the catalogs. Correlations of $\sim 0.4$ arise between $A_{3}$ and $D_{\alpha}$, and of $\sim 0.5$ between $D_{\delta}$ and $B_{\delta}$.

Parameter values are roughly comparable between the three source categories (all, defining, and non-defining), except for aus2013a for which the global orientation of the non-defining sources significantly differs from the orientation of the defining subset. It appears that most of the catalog orientations agree with the ICRF2 within $20 \mu$ as. The catalog aus2013a, however, presents a significant rotation around the $X$-axis that does not show up in other solutions. Similar angles were recently published by Sokolova \& Malkin (2014). The authors also mentioned that the non-inclusion of the full covariance information between all the coordinates of the sources (and not only between right ascension and declination of each source) could be a possible explanation of the still significant rotation angles, even though sources are constrained to be aligned onto the ICRS.

The postfit root mean square (rms; i.e., the rms computed after rotation) is significantly larger than the rotation angles. Nevertheless, postfit rms obtained from Calc/Solve solutions tend to be lower. Since the ICRF2 was also obtained with Calc/Solve, the deviation observed for aus2013a is likely to be attributed to the analysis method used in OCCAM. Although $\chi^{2}$ is reasonably close to unity for the non-defining sources, it appears generally larger than 10 for the defining sources, indicating a possible underestimation of the formal errors as published in the catalogs.

We note that the form of the transformation as expressed in (1) considers the $Z$-axis as a privileged direction. The artificial displacement of the sources towards the poles was already noticed in several earlier works (e.g., Ma et al. 1998; Gontier et al. 2001; Feissel-Vernier 2003). It can be physically understood as resulting from the dissymmetry of the network: during the first two decades of VLBI, most of the antennas (and most of the observed sources) were in the northern hemisphere. Networks and analyses were strongly sensitive to the troposphere thickness on the equator. A more general transformation corresponds to the first degree toroidal and spheroidal harmonics of a vector field (see, e.g., Mignard \& Klioner 2012). In the case of the studied catalogs, both transformations give comparable results.

Then, I checked the ability of the catalogs to define the system axes when only a subset of sources is considered. To achieve this, I computed the standard deviation of the transformation parameters estimated for a thousand of subsets selected randomly. 
Table 2. Systematics and statistics of the difference to ICRF2.

\begin{tabular}{|c|c|c|c|c|c|c|c|c|c|c|c|c|c|c|c|c|}
\hline & \multicolumn{12}{|c|}{ Transformation parameters } & \multicolumn{2}{|c|}{ rms } & \multicolumn{2}{|c|}{$\chi^{2}$} \\
\hline & $A_{1}$ & \pm & $A_{2}$ & \pm & $A_{3}$ & \pm & $D_{\alpha}$ & \pm & $D_{\delta}$ & \pm & $B_{\delta}$ & \pm & $\alpha \cos \delta$ & $\delta$ & $\alpha \cos \delta$ & $\delta$ \\
\hline \multicolumn{17}{|c|}{ All sources } \\
\hline aus2013a & -27 & 5 & 0 & 5 & -2 & 4 & 12 & 5 & 19 & 6 & -10 & 4 & 77 & 77 & 3.1 & 2.6 \\
\hline bkg2013a & -4 & 4 & 12 & 4 & -5 & 3 & 1 & 4 & 12 & 5 & 13 & 3 & 56 & 75 & 5.0 & 4.5 \\
\hline gsf $2012 a$ & -1 & 4 & 6 & 4 & -6 & 3 & -2 & 4 & 16 & 5 & -16 & 3 & 47 & 63 & 4.4 & 4.0 \\
\hline iaa2012a & -8 & 4 & 7 & 4 & -6 & 3 & -11 & 4 & 63 & 5 & -26 & 4 & 57 & 76 & 1.9 & 2.0 \\
\hline opa2013a & -7 & 4 & 12 & 4 & -10 & 3 & 1 & 4 & 5 & 5 & 4 & 3 & 50 & 64 & 4.4 & 4.0 \\
\hline \multicolumn{17}{|c|}{ Defining sources } \\
\hline aus2013a & -21 & 6 & 1 & 6 & -1 & 5 & 14 & 6 & 24 & 7 & -13 & 5 & 63 & 60 & 13.9 & 10.3 \\
\hline bkg2013a & -2 & 5 & 12 & 5 & -4 & 4 & 2 & 5 & 19 & 6 & 12 & 4 & 45 & 55 & 18.6 & 14.3 \\
\hline gsf2012a & -2 & 5 & 7 & 5 & -4 & 4 & -2 & 5 & 19 & 6 & -14 & 4 & 41 & 54 & 20.0 & 17.1 \\
\hline iaa2012a & -6 & 6 & 5 & 6 & -7 & 4 & -7 & 6 & 74 & 7 & -30 & 5 & 44 & 63 & 6.4 & 7.8 \\
\hline opa2013a & -4 & 5 & 13 & 5 & -10 & 4 & 1 & 5 & 9 & 6 & 5 & 4 & 45 & 54 & 21.4 & 16.5 \\
\hline \multicolumn{17}{|c|}{ Non-defining sources } \\
\hline aus $2013 a$ & -41 & 9 & -2 & 8 & -5 & 7 & 8 & 9 & 4 & 11 & 2 & 8 & 125 & 134 & 1.6 & 1.4 \\
\hline bkg2013a & -6 & 7 & 12 & 6 & -8 & 5 & 0 & 6 & 1 & 8 & 16 & 6 & 79 & 109 & 3.1 & 3.1 \\
\hline gsf $2012 a$ & -2 & 6 & 5 & 6 & -9 & 5 & -3 & 6 & 13 & 8 & -19 & 6 & 61 & 81 & 2.2 & 2.1 \\
\hline iaa2012a & -12 & 7 & 8 & 6 & -6 & 5 & -15 & 7 & 46 & 9 & -16 & 6 & 80 & 100 & 1.2 & 1.1 \\
\hline opa2013a & -10 & 7 & 11 & 6 & -11 & 5 & 2 & 6 & 1 & 8 & 1 & 6 & 63 & 85 & 2.0 & 2.1 \\
\hline
\end{tabular}

Notes. Units are $\mu$ as except for $D_{\alpha}$ and $D_{\delta}$ which are in $\mu$ as per radian.

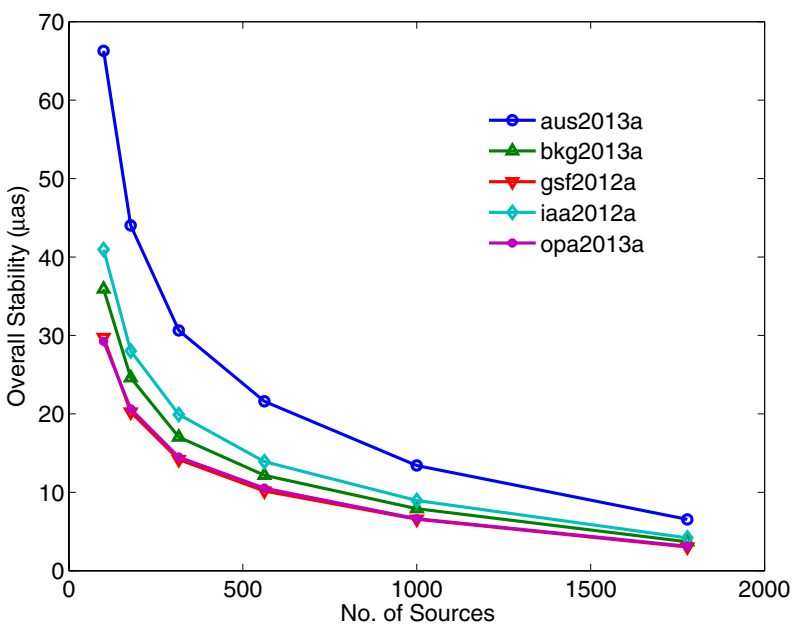

Fig. 2. Stability of the catalogs for subsets of sources of increasing size.

Figure 2 shows the overall stability obtained as the quadratic sum of the standard deviations of the six transformation parameters for subset sizes varying between 100 and about two thirds of the total number of sources. All catalogs have stabilities less than 0.1 mas for more than 100 sources. The stability improves to less than $10 \mu$ as for more than 1500 sources.

\section{Errors and offsets to ICRF2}

Although the ICRF2 is not the truth, it constitutes a reliable representation of the celestial system. For most of sources which had a large observational history when the ICRF2 was released, the addition of new observations should not have changed the estimated position significantly, unless some cataclysmic event happened in the mean time (e.g., strong variation of the flux of an ejected component) and displaced the radio center significantly, or if data is corrupted. In an ideal case, the offsets between a recent catalog and the ICRF2 should not be statistically significant

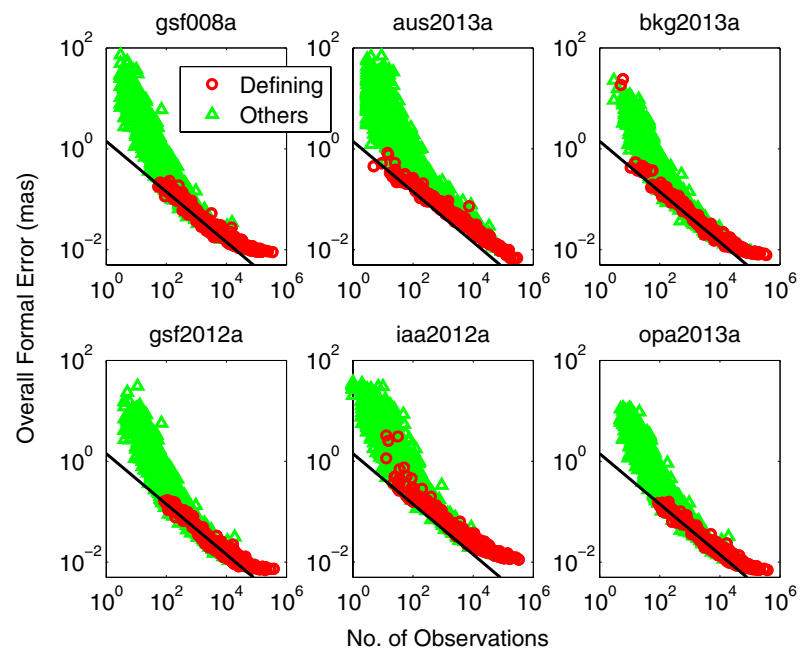

Fig. 3. Dependence of the overall formal error on the number of delays. The black line represents a decrease following $1 / \sqrt{N}$.

and so should be accounted for by the errors provided in the catalog. The $\chi^{2}$ of these differences should therefore be close to 1 . For the purpose of the following analyses, I eliminated sources with offsets to ICRF2 larger than a 1 milliarc second (mas) and ratios of the offset to ICRF2 to the formal error larger than 10.

Figure 3 illustrates how the overal formal error, defined as the square root of $\sigma_{\alpha \cos \delta}^{2}+\sigma_{\delta}^{2}+c \sigma_{\alpha \cos \delta} \sigma_{\delta}$ where the $\sigma$ are the formal errors listed in the catalogs and $c$ is the correlation between estimates of $\alpha$ and $\delta$, varies with the number $N$ of observations. The plot relevant to the gsf008a solution, i.e., the ICRF2 catalog before inflation of the formal errors and before rotation onto the ICRS, is shown for comparison. This solution was processed with Calc/Solve (Fey et al. 2009). In the case of white noise measurements, the formal error is expected to decrease as $1 / \sqrt{N}$. Figure 3 reveals that this regime exists for $N$ between $\sim 100$ and $\sim 10000$ (I considered only sources whose 
Table 3. Noise floor and scale factor. Units are $\mu$ as.

\begin{tabular}{|c|c|c|c|c|c|c|c|c|}
\hline & \multicolumn{4}{|c|}{ Noise floor } & \multicolumn{4}{|c|}{ Scale factor } \\
\hline & $\alpha \cos \delta$ & \pm & $\delta$ & \pm & $\alpha \cos \delta$ & \pm & $\delta$ & \pm \\
\hline aus2013a & 106 & 24 & 97 & 16 & 0.85 & 0.07 & 0.86 & 0.06 \\
\hline bkg2013a & 58 & 6 & 72 & 10 & 1.29 & 0.06 & 1.27 & 0.07 \\
\hline gsf2012a & 57 & 4 & 68 & 10 & 0.82 & 0.05 & 0.77 & 0.08 \\
\hline iaa2012a & 64 & 8 & 87 & 18 & 0.79 & 0.06 & 0.80 & 0.09 \\
\hline opa2013a & 55 & 5 & 63 & 11 & 0.81 & 0.05 & 0.88 & 0.08 \\
\hline
\end{tabular}

coordinates were estimated as global parameters, although catalogs can also contain averaged coordinates of some source time series). Sources having less than a hundred observations were observed in a very few number of sessions. However, most of them were observed by the 10-station VLBA network. The formal error of the coordinates of these sources varies in $1 / N$ in all case. Beyond 10000 observations, the formal error generally tends towards a limit lower than $\sim 10 \mu$ as, as already mentioned in Fey et al. (2009). Such a deviation is visible for all catalogs except aus2013a for which the formal error continues to decreases following $\sim N^{-0.4}$. The deviation for large $N$ is likely the signature of non-Gaussian correlated errors, or station-dependent noise incoming from time- and space-correlated clock and troposphere parameters mismodeling (Gipson 2006; Romero-Wolf et al. 2012). As $N$ increases, thermal baseline-dependent error tends to zero and the station-dependent error becomes dominant. In the future, the correction of this defect should be achieved by better modeling and parameterization of clock and troposphere correlated errors.

Several studies showed evidence that the formal errors of VLBI products (e.g., Earth orientation parameter or station coordinate time series) are underestimated (see, e.g., Ryan et al. 1993). Formal errors can be made more realistic by applying a scale factor $s$ and adding a noise floor $f$ in quadrature, so that the recalculated errors are given by $\sqrt{(s \sigma)^{2}+f^{2}}$. For previous realizations of the ICRF, the noise floor was determined by comparison of various solutions made with subparts of the data set or different softwares and different analysis options. These methods are not applicable here because it would need further reanalyses from all analysis centers.

I tried to get rough estimates of the noise floor and the scale factor by computing the rms scatter of the position offsets to ICRF2 (after applying the systematic differences) binned by interval of formal error for all solutions. A similar method was used by Herring et al. (2002) and Lambert et al. (2008) for rescaling the formal errors of VLBI nutation time series. If the offsets to ICRF2 were accounted for by the formal errors, the scatter would be on the order of the error. If formal errors were underestimated, the scatter would be larger than the error. A noise floor would appear as a limit of the scatter when the error decreases. The nodes of the intervals run from $1 \mu$ as to 1 mas by steps of $40 \mu$ as (Fig. 4). Such an interval allows a sufficient number of points in each bin. The figure shows that formal errors larger than 0.1 mas are associated with smaller scatter. However, for low formal errors, the scatter is clearly larger. The fit yields scale factors generally around 0.8 and noise floors between $50 \mu$ as and $100 \mu$ as (Table 3 ). We note that this noise floor does not strictly represent the internal noise in the catalogs since I considered the differences to the ICRF2: the noise floor necessarily contains a part of the noise incoming from the ICRF2 itself and should not be directly compared with the $40 \mu$ as noise floor of Fey et al. (2009) which was determined with a different method. Nevertheless, assuming the noise floor of the ICRF2
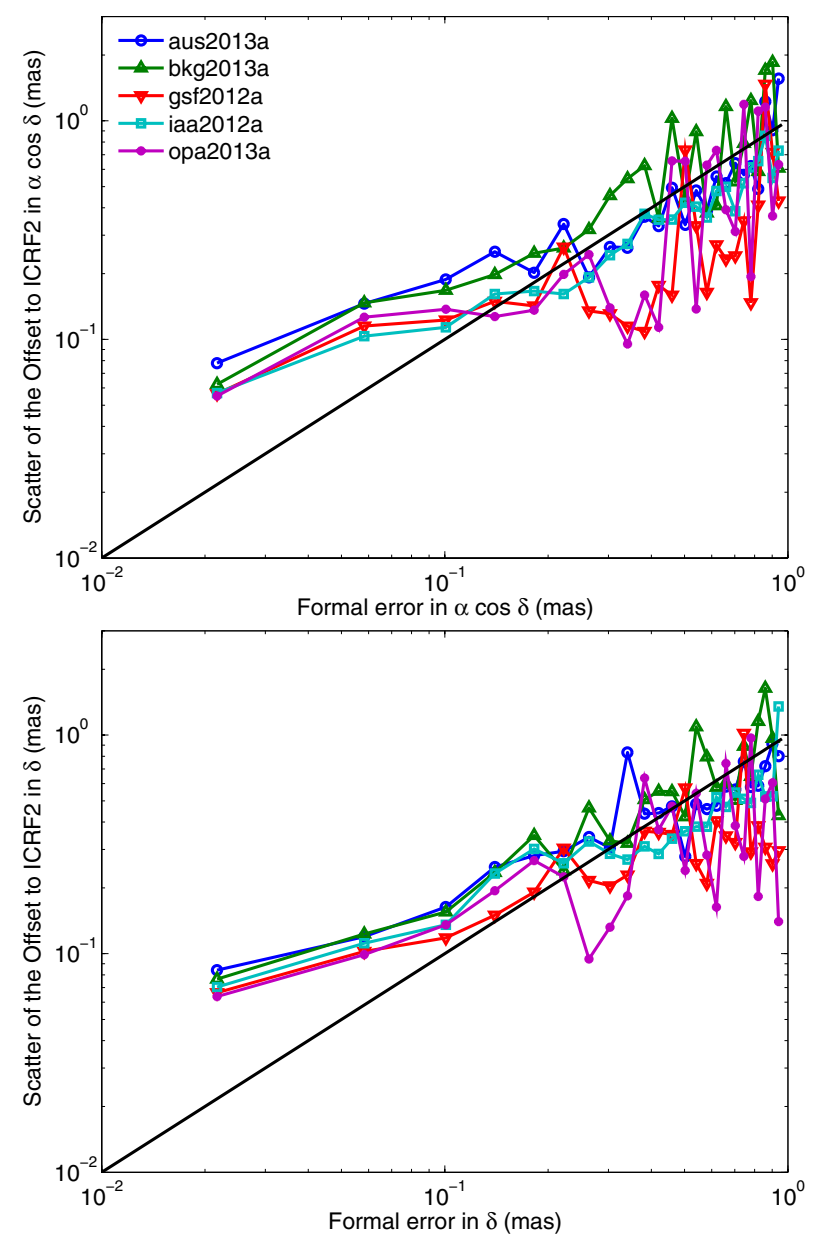

Fig. 4. Scatter of the offset to ICRF2 vs. the formal error.

at $40 \mu$ as leads to individual noise floors on the same order or larger for all catalogs.

\section{Concluding remarks}

I checked the consistency of recently submitted extragalactic radio source catalogs obtained from VLBI analysis. All catalogs are consistent with the ICRF2 within $20 \mu$ as, which is quite satisfactory considering that (i) the ICRF2 axis stability was estimated to $10 \mu$ as in 2009 and (ii) radio source evolution since then may have slightly destabilized the axes (Lambert 2013). The comparison of offsets to ICRF2 against formal errors raised noise floors of the differences between $50 \mu$ as and $100 \mu$ as. This rough evaluation tells us that the accuracy of the catalogs has not improved significantly since the release of the ICRF2. The inconsistencies between catalogs and their accuracy are at the 
level of or larger than the accuracy expected for the future realizations of the celestial reference frame. In the near future and in view of the generation of the next ICRF (Jacobs et al. 2014), it will be necessary to clarify the differences between the various software packages, and to extensively discuss the analysis strategy. In particular, one should understand and correct for the effect of correlated noise.

Acknowledgements. I thank the International VLBI Service for Geodesy and Astrometry (IVS) for their permanent efforts to schedule, observe, correlate, and analyze VLBI data, as well as C. S. Jacobs for useful discussions, and an anonymous referee who helped in improving the manuscript.

\section{References}

Böhm, J., Werl, B., \& Schuh, H. 2006, J. Geophys. Res., 111, B02406 Feissel-Vernier, M. 2003, A\&A, 403, 105

Feissel-Vernier, M., Ma., C., Gontier, A.-M., \& Barache, C. 2005, A\&A, 438, 1141

Feissel-Vernier, M., Ma., C., Gontier, A.-M., \& Barache, C. 2006, A\&A, 452, 1107

Fey, A. L., Gordon, D. G., Jacobs, C. S., et al. 2009, International Earth Rotation and Reference Systems Service (IERS) Technical Note 35, Bundesamts für Kartographie und Geodäsie, Frankfurt am Main

Gipson, J. M. 2006, in International VLBI Service for Geodesy and Astrometry (IVS) 2006 General Meeting Proceedings, NASA/CP-2006-214140, eds. D. Behrend, \& K. D. Baver, 286

Gontier, A.-M., Le Bail, K., Feissel, M., \& Eubanks, T. M. 2001, A\&A, 375, 661
Herring, T. A., Mathews, P. M., \& Buffett, B. A. 2002, J. Geophys. Res., 107 (B4)

Heinkelmann R., \& Tesmer V. 2013, in Reference Frames for Applications in Geosciences, eds. Z. Altamimi, \& X. Collilieux, IAG Symp., 138, 181

IERS 1996, International Earth Rotation Service (IERS) Annual Report 1995 (Observatoire de Paris, Paris), II-19

Jacobs, C. S., Arias, E. F., Boboltz, D., et al. 2014, in Proc. Journées 2013 Systèmes de Référence Spatio-Temporels, Observatoire de Paris, ed. N. Capitaine, 26

Lambert, S. 2013, A\&A, 553, A122

Lambert, S., \& Dehant, V. 2007, A\&A, 469, 777

Lambert, S., Dehant, V., \& Gontier, A.-M. 2008, A\&A, 481, 535

Ma, C., Arias, E. F., Eubanks, T. M., et al. 1998, AJ, 116, 516

Mignard, F., \& Klioner, S. 2012, A\&A, 574, 59

Petit, G., \& Luzum, B. J. 2010, International Earth Rotation and Reference Systems Service (IERS) Technical Note 36, Frankfurt am Main: Verlag des Bundesamts für Kartographie und Geodäsie

Romero-Wolf, A., Jacobs, C. S., \& Ratcliff, J. T. 2012, in International VLBI Service for Geodesy and Astrometry (IVS) 2012 General Meeting Proceedings, NASA/CP-2012-217504, eds. D. Behrend, \& K. D. Baver, 231

Ryan, J. W., Clark, T. A., Ma, C., et al. 1993, in Contributions of Space Geodesy to Geodynamics: Earth Dynamics, (Washington: Am. Geophys. Union), eds. D. E. Smith, \& D. L. Turcotte, 37

Schuh, H., \& Behrend, D. 2012, J. Geodyn., 61, 68

Skurikhina, E., Kurdubov, S., \& Gubanov, V. 2013, in International VLBI Service for Geodesy and Astrometry (IVS) 2012 Annual Report, NASA/TP2013-217511, eds. K. D. Baver et al., 269

Sokolova, Y. R., \& Malkin, Z. M. 2014, Astr. Lett., 40, 268

Titov, O., Tesmer, V., \& Böhm, J. 2004, in International VLBI Service for Geodesy and Astrometry (IVS) 2004 General Meeting Proceedings, NASA/CP-2004-212255, eds. N. R. Vandenberg, \& K. D. Baver, 267 